\title{
Archéopages
}

Archéopages

Archéologie et société

Hors-série 3 | 2012

Nouveaux champs de la recherche archéologique

\section{Mal enterrés et mal rangés : le cas des squelettes humains sans sépulture en France gallo-romaine et médiévale}

Isabelle Rodet-Belarbi et Isabelle Séguy

\section{(2) OpenEdition}

Journals

Édition électronique

URL : https://journals.openedition.org/archeopages/719

DOI : 10.4000/archeopages.719

ISSN : 2269-9872

Éditeur

INRAP - Institut national de recherches archéologiques préventives

Édition imprimée

Date de publication : 1 janvier 2012

Pagination : 168-172

ISSN : 1622-8545

\section{Référence électronique}

Isabelle Rodet-Belarbi et Isabelle Séguy, « Mal enterrés et mal rangés : le cas des squelettes humains sans sépulture en France gallo-romaine et médiévale », Archéopages [En ligne], Hors-série 3 | 2012, mis en ligne le 01 janvier 2012, consulté le 25 février 2023. URL : http://journals.openedition.org/ archeopages/719; DOI : https://doi.org/10.4000/archeopages.719 


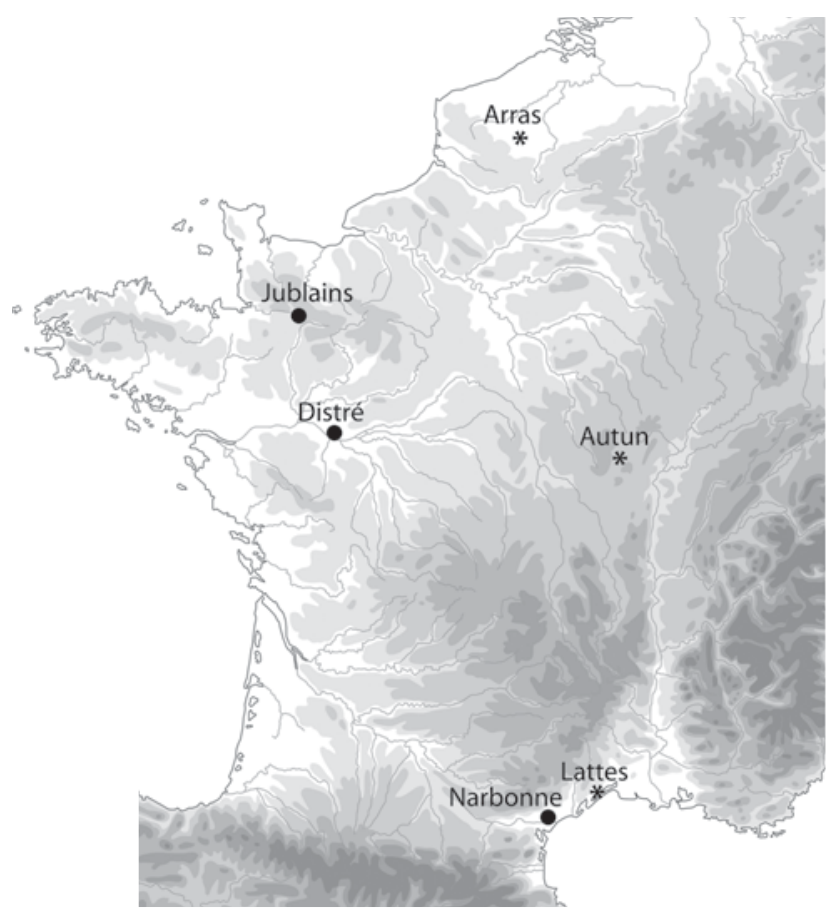

- Sites avec puits et fosses contenant exclusivement des périnataux dans leurs comblements : Narbonne (Aude; courant $\mathrm{III}^{\mathrm{e}} \mathrm{s}$.), Jublains (Mayenne ; fin $\mathrm{III}^{\mathrm{e}}$-début $\mathrm{IV}^{\mathrm{e}} \mathrm{s}$.) et Distré (Maine-et-Loire ; $\mathrm{VI}^{\mathrm{e}} \mathrm{s}$.)

* Sites avec puits et fosses contenant différents groupes d'âge dans leurs comblements : Lattes

(Hérault; dernier quart du $\mathrm{I}^{\mathrm{er}} \mathrm{s}$.), Autun (Saône-et-Loire ; fin $\mathrm{II}^{\mathrm{e}} \mathrm{s}$.- début $\mathrm{III}^{\mathrm{e}} \mathrm{s}$.) et

Arras (Nord; $\mathrm{IV}^{\mathrm{e}} \mathrm{s}$.)
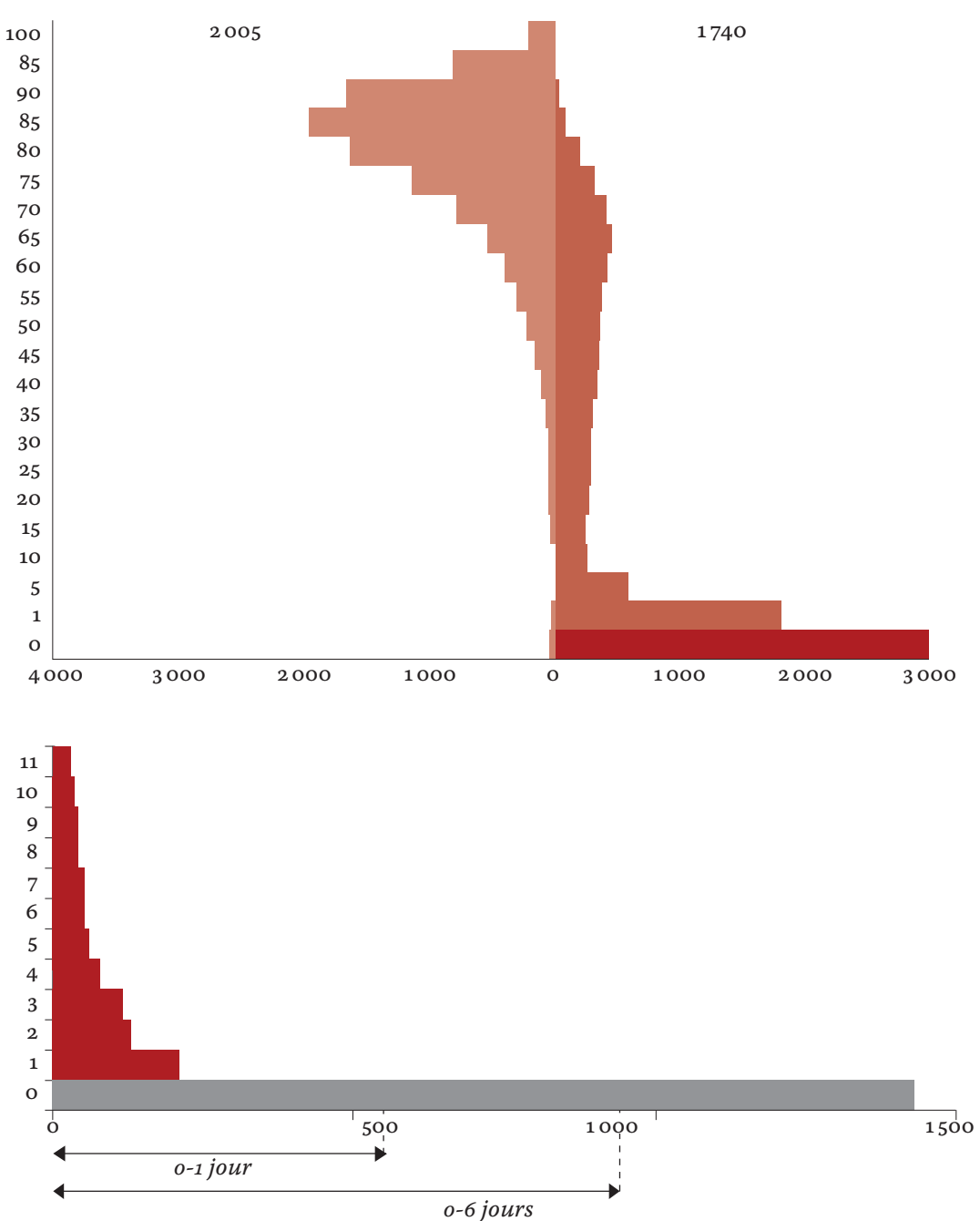

[Fig 1] Localisation des sites mentionnés dans le texte.
[Fig.2] Distribution proportionnelle des décès par âge en France, en 1740 (à droite) et en 2005 (à gauche).

La démographie des population préindustrielles est très différente de l'actuelle ainsi qu'on peut le constater, ici, à travers le poids de la mortalité à certains âges : autrefois, un très grand nombre d'enfants décédaient avant leur $5^{\mathrm{e}}$ anniversaire ; cela résultait à la fois d'une forte fécondité et d'un risque de mourir très important entre o et 4 ans, et maximal avant 1 an. (Source : Blayo, 1975 et INSEE)
[Fig.3] Détail de la distribution des décès entre o et 1 an, d'après la table de mortalité dressée par R. Lalou (1990) pour la population canadienne du XVIII ${ }^{\mathrm{e}} \mathrm{s}$. Parmi les enfants qui ne survivaient pas jusquà leur premier anniversaire (220o dans cet exemple), près de la moitié (920) décédaient avant la fin de leur première semaine de vie ; près du quart $(520)$ ne vivaient que quelques heures (o-1 jour) 
du Mouraut du peuplement de l'Aquitaine gothe à la veille de la bataille de Vouillé (507), sont d'ores et déjà majeurs. Ils éclairent de façon nouvelle certains aspects des modalités d'installation et d'intégration des nouveaux venus et obligent à intégrer la présence avérée, au cœur du regnum, de groupes humains culturellement homogènes (et non plus seulement d'individus ou de «familles»), de véritables communautés non romaines implantées en milieu rural : les Goths.

вӧнмЕ H.W., 1974 : Germanishe Grabfunde des 4. Bis 5. Jahrunderts zwischen unteren Elbe und Loire, Beck, Munich, 2 vol.

BOUDARTCHOUK J.-L., 1998 : «La nécropole franque d'Ictium à L'IsleJourdain (Gers, Midi-Pyrénées) », in Die Franken - Les Francs, Acta Praehistorica et Archaeologica, 3o, Berlin, p. 126-136.

JAMES E., 1977 : The merovingian archaeology of south-west Gaul, British Archaeological reports, Supplementary series 25, Oxford, 2 vol.

KAZANSKI M., 2010 : «Les Wisigoths, du Danube à la Gaule (375-412)» in Bourgeors L. (DIR.) Wisigoths et Francs autour de la bataille de Vouillé (507): Recherches récentes sur le haut Moyen Age dans le Centre-Ouest de la France, Actes des XXVIII journées internationales d'archéologie mérovingienne, Saint-Germain-enLaye, p. 9-14.

Rouche M., 1979: L'Aquitaine des Wisigoths aux Arabes 418-781: naissance d'une nation, École des Hautes Études en Sciences Sociales, J. Touzot (éd.), Paris, 778 p.

Wolfram H., 1991 : Histoire des Goths, Albin Michel, coll. Lévolution de l'humanité, Paris, 574 p.

\section{Mal enterrés et mal rangés : le cas des squelettes humains sans sépulture en France gallo-romaine et médiévale}

Isabelle Rodet-Belarbi

Inrap, UMR 7264 «Cultures-Environnements, Préhistoire, Antiquité, Moyen Âge »

Isabelle Séguy

INED, UMR 7264 «Cultures-Environnements, Préhistoire,

Antiquité, Moyen Âge »

$\mathrm{L}$

es nombreuses fouilles en contextes archéologiques variés, couvrant de larges espaces et de longues périodes chronologiques, ont permis la découverte de vestiges très différents les uns des autres, dont certains témoignent de situations peu communes. L'une d'elles illustre un phénomène connu par les textes médiévaux ${ }^{\mathbf{1}}$ : celui des enfants mort-nés ou dont on voulait se débarrasser dès la naissance et qui ne recevaient aucune sépulture.

Ainsi, lorsque Cécile Treffort se demande à quoi correspondent les « corps abandonnés sans soin particulier à la merci des animaux sauvages ou

1 P.-A. Sigal mentionne le cas d'une habitante d'Aix-en-Provence ayant accouché de jumeaux mort-nés dont les corps furent jetés sur un tas de fumier (Sigal, 1987, p. 31) Des procès médiévaux et modernes relatent des cas d'infanticides ; les corps des nouveaunés ayant été jetés dans des latrines, des puits, des auges à cochons... 2 Le terme «périnatal » regroupe les enfants morts juste avant, pendant ou juste après leur naissance. à notre mantalité dur. Bien que cette «idée répugne par des témoignages médiévaux (Treffort, 1997, p. 101). En effet, « les pénitentiels du Haut Moyen Âge interdisent formellement de consommer des animaux, y compris des bêtes délevage, ayant mangé de la chair humaine. On peut se demander, avec P. Bonnassie, à qui appartiennent ces corps. S'agit-il entre autres, se demande-t-il, «d'enfants excédentaires, délibérément "perdus", de nouveau-nés exposés » ou, pourrions-nous ajouter, d'enfants morts en bas âge et non ensevelis ? (Treffort, 1997, p. 102).

Des découvertes archéologiques montrent que, si certains nouveau-nés sont enterrés dans les nécropoles ou les cimetières, d'autres le sont au sein des habitats, dans des sépultures inhabituelles. Il en est d'autres encore qui, manifestement, n'ont eu droit à aucun égard et ont été jetés parmi les rejets domestiques. Nous en avons des exemples qui, par leur similitude, nous ont permis de proposer la notion de «non-sépulture» (Rodet-Belarbi, Séguy, à paraître ; Séguy, Rodet-Belarbi, à paraître) et de répondre par l'affirmative au questionnement des historiens. En effet, des squelettes de périnataux ${ }^{2}$ ont été jetés dans des dépotoirs, des décharges, des puits en cours de comblement... et mis au jour au beau milieu des lots de faune. Comme il s'agit d'individus complets, et non d'os erratiques résultant de l'écrêtage de nécropoles et de cimetières antérieurs, nous y avons vu un geste volontaire. Il était nécessaire de disposer de la double détermination homme/ animal pour que notre analyse ne soit pas biaisée et repose sur des informations exhaustives. Ce faisant, nous nous sommes aperçu que ce phénomène n'était pas exclusivement réservé aux périnataux, mais que d'autres groupes d'âges pouvaient être concernés. Un tel phénomène est illustré par diverses découvertes en France.

Les individus périnataux. Deux sites éloignés géographiquement, mais proches chronologiquement, Jublains (Mayenne ; Bocquet et al., 2004) et Narbonne (Aude; Sabrié, 2011), présentent des caractéristiques communes [Fig.1]. Sur ces deux sites, occupés dès le début du I ${ }^{\text {er }}$ siècle de notre ère, des puits à eau ont été creusés au sein d'un quartier artisanal, dans le premier cas, et au centre d'une domus, dans le second.

Lors de son abandon, daté de la fin du III ${ }^{\mathrm{e}}$-début du IV ${ }^{\mathrm{e}}$ siècle, le puits PT 3082 de Jublains a été essentiellement comblé de déchets artisanaux, de restes osseux correspondant à des reliefs de repas, de squelettes animaux et humains. Des cadavres de chiens de tous âges, de deux chèvres, d'un équidé adulte, ainsi que les ossements d'un périnatal âgé de 9-10 mois lunaires ont été identifiés (Forest, Fabre [coll.], 1999, p. 8). Malgré un bon état de conservation et le tamisage des sédiments de la moitié inférieure du remplissage, les os humains et animaux de petite taille n'ont pas été retrouvés.

Le puits de la maison au grand Triclinium de Narbonne, abandonné au début du III ${ }^{\mathrm{e}}$ siècle et comblé au cours du même siècle, a été rempli de déchets divers : céramiques, détritus alimentaires et os erratiques de diverses espèces consommées (porc, mouton/chèvre, cerf, lapin de garenne, oiseaux et poissons). Comme à Jublains, divers squelettes y ont été découvert : chiens immatures et adultes, jeune équidé, singe magot (Macaca sylvana) et aussi enfants morts en période périnatale (Forest, Fabre, 2011). À nouveau, malgré l'absence de leurs os les plus petits, tout porte à croire que ces squelettes humains étaient complets lors de leur rejet dans le puits. Les os rochers ont permis d'estimer à 27 le nombre minimum d'individus. Ce chiffre peut paraître très important au regard de la séquence d'utilisation de ce puits comme dépotoir, qui n'a duré que quelques

dizaines d'années dans un quartier en cours 
d'abandon. Il n'est toutefois pas incompatible avec les hauts niveaux de mortalité des enfants aux époques préindustrielles. En effet, en moyenne, un enfant sur trois mourait avant son premier anniversaire [Fig. 2] et un enfant sur dix dans les heures qui suivent sa naissance [Fig. 3]. Ajouté à un nombre important d'enfants mort-nés (environ $5 \%$ des naissances vivantes), la mortalité périnatale était de l'ordre de 15\% (Séguy, 2011).

Les adultes. Des squelettes d'adultes, parmi des lots de faune, sont également attestés dans au moins deux agglomérations gallo-romaines, situées chacune à une extrémité de la France. Dans le premier cas, il s'agit du site de Lattes (Hérault), où de nombreux puits ont été fouillés dans le quartier sud-est de cette cité portuaire. Lors de leur abandon, ils ont été comblés par divers rejets et, dans le remplissage de deux d'entre eux, des squelettes d'adultes et d'un enfant ont été mis au jour. Le premier puits PT 129011, utilisé comme puits à eau entre 25 et 75 de notre ère, est ensuite désaffecté au cours du dernier quart du ${ }^{\mathrm{er}}$ siècle.

Il a alors servi de dépotoir recevant des squelettes entiers d'équidés, de porcs, de chiens et de lapins, des déchets de boucherie bovine et de rejets de repas. Y ont également été découverts les squelettes de deux hommes adultes, dont l'âge au décès a été estimé par les anthropologues à 30-40 ans, pour l'un, et 50-60 ans, pour l'autre. Leurs ossements portent des traces de lésions anciennes et de fractures peri-mortem. "Ces individus ont certainement été jetés simultanément, et sans doute violemment, dans le puits. » (Duchesne, Treil, 2005, p. 338). Le second puits PT 471, comblé durant la même période chronologique, a livré le même type de remplissage. Celui-ci contenait un seul squelette d'adulte, âgé de 40-50 ans, dont les os du nez, des bras, des avant-bras et les côtes gardent la trace de nombreux traumatismes résultants de coups violents. En outre, le fémur gauche présente une fracture peri-mortem (Duchesne, Treil, 2005, p. 336). Cet adulte de sexe masculin est " accompagné » d'un périnatal âgé d'environ 10 mois lunaires qui, lui, ne témoigne d'aucune pathologie particulière.

Le cas du site de la rue Baudimont à Arras (Nord) est un peu différent. Une des fosses a été interprétée, en raison des nombreux ossements de bœufs découverts dedans, comme un dépotoir de boucherie exploité au IV ${ }^{\mathrm{e}}$ siècle. Mêlés à ces déchets, quatre squelettes humains et celui d'un chien ont été retrouvés « empilés et enchevêtrés » (Blondiaux, 1990, p. 95). Il s'agit des squelettes complets de deux enfants, l'un âgé de 8 ans et l'autre de 9-10 ans, et de deux adultes de sexe masculin, l'un jeune, l'autre, plus âgé. Ce dernier présente « un tassement vertébral d'origine accidentelle probable ». Aucune trace de violence, aucune fracture peri-mortem susceptible d'éclaircir les circonstances de leur mort n'a été repérée. Cependant, l'anthropologue préfère, à l'hypothèse de cadavres jetés suite à une famine ou à une épidémie, celle du « massacre d'hommes d'une même famille » (Blondiaux et al., 1991, p. 18).
La communauté scientifique face à ces associations insolites. Dans les quatre cas précités, les anthropologues et les archéozoologues sollicités ont mené leurs études parallèlement ou de conserve. Leur traitement des données et leurs réactions face à ces ossements humains mêlés à d'autres détritus sont diverses. Les positions éditoriales, souvent plus enclines aux partitions classiques par sujet d'étude, s'ouvrent peu à peu à des présentations mixtes permettant l'émergence et la valorisation d'une thématique peu commune.

Ainsi, les squelettes humains d'Arras ont fait l'objet de traitements différents dans deux publications. Dans le catalogue de l'exposition Les cultes à Arras au Bas Empire, les études archéozoologique et anthropologique du site de Baudimont sont distinctes. La partie relative à l'archéozoologie ne traite pas du tout la fosse de boucherie, tandis que l'étude anthropologique ne souligne pas l'originalité de la découverte de quatre corps dans ce dépotoir de boucherie (Blondiaux, 1990). Dans la seconde publication, non plus, aucun commentaire particulier ne suit la description des découvertes : «Il s'agit en fait d'une fosse de boucherie remplie de déchets animaux. Elle contenait deux squelettes complets d'adultes (Foo2 et Foo3), deux squelettes d'enfants (Foo1 et Foo4), empilés les uns sur les autres, en désordre, et habillés. » (Blondiaux et al., 1991, p. 12).

Pourtant, les termes employés, « empilés » et « en désordre », sont sans équivoque sur le fait que ces personnes n'ont reçu aucune sépulture.

À Lattes, lors des fouilles menées sous la direction de Michel Py entre 1986 et 200o, Véronique Fabre (anthropologue) et Armelle Gardeisen (archéozoologue) ont assuré le tri des ossements issus des onze puits explorés (Piquès, Buxó, 2005, p. 350). Les restes humains ont été confiés pour étude à Sylvie Duchesne et Jacques Treil, tandis que les animaux étaient répartis entre divers spécialistes selon qu'il s'agissait de mammifères, de poissons, d'oiseaux... Toutes leurs études ont été publiées dans des chapitres thématiques différents, réunis au sein d'un même ouvrage, qui permet au lecteur d'accéder directement à ses centres d'intérêts. Toutefois, les archéozoologues, dans le cas du puits PT 129011, font remarquer « la présence de deux squelettes humains parmi le mobilier osseux » (Valenzuela Lamas, Gardeisen, 2005, p. 240). Une mention similaire est faite pour le squelette d'humain adulte du puits PT 471. De leur côté, les archéologues et les anthropologues ont échangé leurs informations et leurs remarques montrent à quel point la découverte d'ossements humains dans le comblement des puits a été troublante. Les archéologues soulignent l'insolite de la découverte, « [...] un squelette humain jeté visiblement au même titre que ces animaux » (Buxó et al., 2005, p. 59). Les anthropologues évoquent que « le fait de les retrouver dans le dépotoir d'un puits, dépôt peu courant, laisse suggérer un traitement particulier et inhabituel pour ces hommes » (Duchesne, Treil, 2005, p. 343). En conclusion, les archéologues, alertés par les constats des anthropologues, posent 
clairement la question du statut social de ces individus : « Les humains semblent avoir été traités au même titre que les animaux, car leur dépôt est précédé et succédé de rejets d'éléments de dépotoir qui n'ont apparemment rien de rituel. Ce sort était-il destiné à une catégorie de la population exclue des pratiques funéraires normales ? Est-ce le résultat d'évènements tragiques ou violents? (Piquès, Buxó, 2005, p. 351) ». Ils notent, en outre, que les mauvais traitements subis par ces hommes et leurs mauvaises conditions de vie sont « en opposition assez nette avec la vision idyllique que l'on a souvent de la société gallo-romaine ».

La seule publication qui regroupe en un seul chapitre les études relatives aux os humains et animaux est celle du puits de Narbonne. Les deux auteurs, Véronique Fabre et Vianney Forest, ont en effet tenu à présenter les résultats ensemble, afin d'insister sur cette position très particulière des restes de périnataux humains au milieu de ceux de chiens, et d'autres ossements d'animaux, de reliefs de repas et de détritus en tous genres. Cette décision a été maintenue contre l'avis d'un premier comité de lecture ${ }^{\mathbf{3}}$ qui suggérait de séparer en deux chapitres les résultats des études, comme cela se fait « classiquement ». L'argument était que cette démarche très novatrice permettait d'alerter clairement sur la présence conjointe d'ossements humains et d'ossements animaux en contexte de dépotoir. Leurs conclusions ciblent d'autant plus l'aspect insolite de « cette série d'au moins 27 enfants périnataux [qui] laisse libre cours à de multiples explications, tout en posant la question générale du statut réel des fotus ou des enfants nouveau-nés dans la société romaine » (Forest, Fabre, 2011, p. 308). Ces deux mêmes spécialistes avaient antérieurement analysé les restes osseux issus du puits de Jublains en 1999, mais sans pouvoir obtenir la même approche en raison de contraintes éditoriales. Dans cet article (Bocquet et al., 2004), le paragraphe concernant la faune est très court et la découverte des ossements humains est totalement passée sous silence. En revanche, dans le rapport de fouilles (Forest, Fabre [collab.], 1999), l'analyse des restes humains fait suite à celle des oiseaux, terminant ainsi la liste des espèces retrouvées dans le puits. Véronique Fabre conclut son rapport ainsi : «La découverte des restes humains en milieu non funéraire et a fortiori dans des puits parmi les déchets artisanaux, culinaires, et autres, est hautement interrogative » (Forest, Fabre [collab.], 1999, p. 8). Elle envisage plusieurs hypothèses : une sépulture domestique détruite lors du réaménagement de l'habitat, un nouveau type de traitement d'un jeune enfant autre que les inhumations proches des habitats, ou un possible infanticide que l'on aurait essayé de dissimuler : « de se débarrasser sans autre préambule d'un cadavre jugé sans intérêt ». Toutefois, l'auteur termine sur une suggestion moins choquante ou plus moralement acceptable : « [...] cette présence répond-elle à une pratique funéraire ou cultuelle particulière?».
La rareté de ces associations, fait avéré ou biais de la recherche scientifique? Lors des opérations archéologiques de sauvetage, les tâches sont réparties de telle sorte que le tri du mobilier, après lavage, est effectué différemment selon la nature des structures découvertes. Si la présence d'une nécropole ou d'un cimetière est reconnue lors du diagnostic, un ou plusieurs anthropologues participent à la fouille et une attention particulière est portée aux ossements humains. En revanche, celle-ci n'est évidemment pas aussi forte sur les sites où on ne s'attend pas à trouver des ossements humains, qu'ils soient erratiques ou qu'ils correspondent à un ou plusieurs individus complets. Si le responsable du tri du mobilier a quelques connaissances anatomiques, il regroupera les os humains et les signalera dans un inventaire. Si ce n'est pas le cas, le lot d'ossements (hommes et animaux indifférenciés) est confié pour étude à un archéozoologue. Ce spécialiste peut en référer de diverses façons : soit les ignorer purement et simplement, et rendre à l'archéologue des sacs où le matériel reste mélangé. Soit noter, en marge des inventaires de faune, la présence d'ossements humains, puis les transmettre à l'archéologue dans des sacs clairement séparés de la faune. Soit, dans le meilleur des cas, l'archéozoologue reporte la quantité de restes humains retrouvée dans les tableaux de comptage, et/ou dans un petit paragraphe en fin d'étude avec une phrase sibylline comme : «Afin d'être exhaustif, signalons également la présence de...». Bien qu'ainsi identifiés, ces ossements humains ne sont pas étudiés, dans une grande majorité des cas, faute d'avoir prévu une ligne budgétaire « anthropologie » pour ces sites où le diagnostic préalable n'avait pas révélé de zone funéraire. Faute aussi de pouvoir rallonger le temps d'étude qui leur a été imparti.

Bien conscientes des biais générés par cette organisation du travail et persuadées que d'autres exemples d'association homme/animal ont été notés à l'occasion d'études de faune, nous en avons recherché des mentions dans les rapports des archéozoologues rendus ces vingt dernières années. Il s'agissait en particulier de compléter nos premières observations sur les non-sépultures et d'étoffer notre échantillon pour les périodes médiévales. Nous en avons trouvé quelques-unes et nous aimerions en citer ici deux exemples : les sites de Distré (Maine-et-Loire, Valais 2002) et d'Autun (Saône-et-Loire). Distré constitue la parfaite illustration du processus décrit précédemment. Dans son rapport d'étude, l'archéozoologue Jean-Hervé Yvinec a dénombré 84. ossements de fotus humains mêlés à la faune du silo 304, dont le remplissage est daté des $\mathrm{VI}^{\mathrm{e}}$ $\mathrm{VII}^{\mathrm{e}}$ siècles (Yvinec, Frères, 1997). Ces ossements ont été reportés dans les tableaux d'inventaire des espèces et conditionnés séparément de la faune. L'archéologue Alain Valais en a rendu compte dans le document final de synthèse en 1997 (Valais et al., 1997). L'affaire en serait peut-être restée là, sans l'exposé de notre recherche à Alain Valais et JeanHervé Yvinec qui en ont immédiatement saisi 3 Communication
personnelle des auteurs. 
l'intérêt et nous ont aimablement fourni tous les compléments d'informations utiles à notre problématique. Le site de Distré entre clairement dans ce que nous avons proposé de définir comme des cas de non-sépultures. La « redécouverte» de ces ossements de périnataux « mal rangés » a eu pour effet de les classer parmi les ossements humains. Un projet de monographie de site est en cours et, si un budget se voit accordé à l'étude anthropologique, ils seront alors étudiés en même temps que les squelettes de la nécropole. Une approche réellement pluridisciplinaire aura ainsi « Sauvé » ce périnatal du double oubli où il se trouvait, ni correctement inhumé, ni correctement classé.

En 1993, lors de l'étude de la faune mise au jour dans le remplissage du seul puits découvert sur le site du lycée militaire à Autun (Saône-et-Loire ; fin $\mathrm{II}^{\mathrm{e}}$-début $\mathrm{III}^{\mathrm{e}}$ siècle ; Chardron-Picault, Pernot, 1999), la présence de 98 os humains a été notée en marge de nos inventaires. En l'absence de ligne budgétaire prévue pour l'anthropologie sur cette opération, cette mention n'a jamais été exploitée. Mais, gardée en mémoire, elle a naturellement trouvé sa place dans cette recherche et une démarche a été effectuée pour pouvoir étudier les ossements humains ${ }^{4}$. Luc Buchet ${ }^{\mathbf{5}}$ a déterminé la présence d'au moins deux adultes, deux jeunes et un périnatal, chacun représenté par quelques os seulement. Près de la moitié des ossements n'a pu être rattachée avec certitude à l'un de ces cinq squelettes, ce qui laisse envisager un nombre minimum d'individus plus important. En outre, le puits n'ayant pas été fouillé dans sa totalité pour des raisons de sécurité, il est impossible de savoir s'il s'agit de restes humains erratiques ou de squelettes complets dont les ossements auraient été disséminés.

"Mal-enterrés" mais bien rangés! Les cas de Distré et d'Autun sont peut-être les premiers d'une série car, une fois les différents acteurs de l'archéologie dûment alertés, d'autres lots de faune renfermant des squelettes humains pourraient être repérés. Et une simple détermination anthropologique permettrait de confirmer rapidement si le cas considéré entre, ou non, dans notre problématique de la non-sépulture, et s'il est nécessaire d'en approfondir l'examen ostéologique.

Certains sites archéologiques paraissent confirmer l'adage « l'on ne reconnaît que ce que l'on connaît »; en particulier celui de Lattes, où les fouilles des puits, entreprises entre 1970 et 1984 sous la direction de Henri Prades, n'ont pas livré le moindre ossement humain, malgré le tamisage des sédiments. Sensibilisés par leur propre expérience, Gaël Piquès et Ramon Buxó concluent prudemment, pour les puits anciennement fouillés, que «bien qu'aucun reste humain ne soit signalé parmi la faune, leur absence ne peut pour le moment être affirmée, en particulier pour les nouveau-nés » (Piquès, Buxó, 2005, p. 350).

Un important travail de sensibilisation doit être entrepris au sein de la communauté archéologique, tant au niveau des opérations de terrain que des études post-fouilles, pour promouvoir l'étude, l'analyse et la publication de ces situations peu banales, à la limite du « religieusement » correct. Non, tous les morts n'étaient pas inhumés dans les cimetières et les nécropoles, certains sont à rechercher dans des lieux plus improbables.

BLAYO Y., nov. 1975 : « La mortalité en France de 1740 à 1829 ", Population, numéro spécial, p. 123-142.

BLONDIAUX J., 1990 : « Les ossements humains du IV ${ }^{\mathrm{e}}$ s. de la rue Baudimont à Arras : histoire de famille? ", in Les cultes à Arras au bas Empire, Catalogue d'exposition, 1990, Musée des Beaux-Arts d'Arras, Service Archéologique de la ville d'Arras, p. 94-95.

BlondiauX J., JACQUES A., BAYARD D. : 1991, « Dix-neuf squelettes dans l'habitat. Énigme dans la ville d'Arras au bas Empire et dans le fisc royal de Berry-au-Bac (o2) au IX siècle », in BUCHET L. (DIR.), Villes et campagnes en Europe Occidentale ( $V^{e}-\mathrm{XIII}^{e}$ siècles), Paris, CNRS (Dossier et documentation archéologique, 14), p. 12-18.

Bocquet A., Chuniaud K., Naveau, J., Dieudonné-Glad N. (collab.), Forest V. (Collab.), Morin S. (collab.), Mortreau M. (COLlAB.), 2004: « Le quartier antique de la Grande-Boissière à Jublains (Mayenne) », Revue archéologique de l'Ouest, 21, p. 131 -174.

Buxó R., PiQUÈs G. VIDAL A., 2005 : « Structure et stratigraphie des puits de Lattes », in PIQuÈs G., Buxó R., p. 31-69.

Chardon-Picault P., Pernot M. (Dir.), 1999 : Le site du Lycée Militaire d'Autun (Saône et Loire) : un quartier gallo-romain d'artisanat métallurgique, Documents d'Archéologie Française, Paris, éd. Maison des sciences de l'Homme, $\mathrm{n}^{\circ} 76$.

DuChesnes S., Treil J., 2005 : « Analyse de trois squelettes humains et de restes de nouveau-nés », in PIQUÈs G., Buxó R., p. 335-344.

Forest V., FABRE V. (COLLAB.), 1999 : Étude archéozoologique. La Grande Boissière-Jublains (Mayenne). Comblement du puits 3082, (15o-25o ap. J.-C.), Rapport tapuscrit, Service départemental du patrimoine, Conseil général de la Mayenne, 13 p., 12 tab., 5 fig., 1 annexe

Forest V., FABre V., 2011, « Les vestiges anthropologiques et zoologiques », in SABRIÉ M., SAbriÉ R. (DIR.), La maison au grand Triclinium du Clos de la Lombarde à Narbonne, Montagnac, éd. Momique Mergoil (Histoire et archéologie romaine, 19), p. 295-310.

LALOU R., 1990 : Des enfants pour le paradis. La mortalité des nouveaunés en Nouvelle France. Université de Montréal (Démographie, Coll. Thèses et Mémoires, $\mathrm{n}^{\circ} 28$ ).

PIQUÈs G., BuXó R., 2005: Onze puits gallo-romains de Lattara ( $I^{\text {er }}$ s. av. $n$.è.-II s. de n.è.) Fouilles programmées 1986-20oo, Lattara, 18, Lattes, ADALR.

RODET-BELARBI I., SÉGUY I., à paraître : « La non-sépulture : Essai de définition et présentation de quelques exemples, de l'Antiquité à la fin du Moyen Âge ", in Guy H., Jeanjean A. (DIR.), Le cadavre en procès, Journée d'étude, 25 mai 2011, Marseille.

SAbrié M., SAbriÉ R. (DIR.), 2011 : La maison au grand Triclinium du Clos de la Lombarde à Narbonne. Montagnac, éd. Momique Mergoil (coll. Histoire et archéologie romaine, 19).

SÉGUY I., 2011 : « La part des anges : évaluation quantitative de la mortalité des premiers jours dans les populations préindustrielles », in Coste M.-C. (DIR.), Le Corps des anges, Journée d'étude sur les pratiques funéraires autour de l'enfant mort au Moyen Âge, Blandyles-Tours, 14 novembre 2009, Milan, Silvana Editoriale, Conseil Général de Seine-et-Marne, p. 124-131.

SÉGUY I., RODET-BELARBI I., à paraître : « La non-sépulture : acte intentionnel ou accident? Essai de caractérisation à partir d'ensembles "zoo-anthropologiques" des périodes historiques (France) », Actes du colloque En Torno a la muerte: Ritos, prácticas, imágenes y discursos: Africa-América-Asia-Europa, Mexico, 5-8 juillet 2011.

SigAL P.-A., 1987 : « La grossesse, l'accouchement et l'attitude envers l'enfant mort-né à la fin du Moyen Âge, d'après les récits des miracles ", in Santé, médecine et assistance au Moyen Âge, Actes du $110 e$ congrès des Sociétés savantes, Montpellier, 1985, Paris, CTHS, t. 1, p. 23-41.

TReffort C., 1997 : « Archéologie funéraire et histoire de la petite enfance. Quelques remarques à propos du Haut Moyen Âge », in FosSIER R. (DIR), La petite enfance dans l'Europe médiévale et moderne, Toulouse, presses universitaires du Mirail, p.93-108.

VALAIs A., 2002 : « Le village carolingien des Murailles (49)», Archéologia, $\mathrm{n}^{\circ} 386$, p. 58-66.

Valais A., Carcaud N., Mistrot V., Morera I., Pradat B., Ruas M.-P., 1997 : Distré « Les Murailles » (Maine-et-Loire), DFS de sauvetage urgent, AFAN, Service Régional de l'Archéologie des Pays de la Loire, 3 t.

VAlenZuela Lamas S., GARdeisen A., 2005 : « L'environnement animal urbain à l'époque gallo-romaine. Le témoignage des trois puits de lattes (PT129011, PT 471 et PT 290) », in PiQuès G., Buxó R., p. 235-270.

YVINEC J.-H., FrÈrES S., 1997 : «Étude archéozoologique du site de Distré "Les Murailles" », in VALAis A. et al., Rapport d'étude Afan/ SRA Pays de la Loire.
4. Merci à Yannick

Labaune et Angélique Tisserand (Service archéologique de la ville d'Autun, Centre municipal de

l'archéologique et du patrimoine Alain Rebourg) de nous avoir autorisée à reprendre les os pour examen. 5 Anthropologue UMR 6130 " CEPAM », Nice, que nous remercions pour ces informations 